\title{
Cyst of the Canal of Nuck in adult females: A case report and systematic review
}

\author{
ANASTASIA PRODROMIDOU ${ }^{1}$, ANNA PASPALA ${ }^{2}$, DIMITRIOS SCHIZAS ${ }^{3}$, \\ ELEFTHERIOS SPARTALIS ${ }^{4}$, CONSTANTINOS NASTOS ${ }^{2}$ and NIKOLAOS MACHAIRAS ${ }^{2}$
}

\author{
${ }^{1}$ Department of Surgery, Division of Surgical Oncology, Metaxa Cancer Memorial Hospital, Piraeus 18573; \\ ${ }^{2}$ Third Department of Surgery, Attiko University Hospital, National and Kapodistrian University of Athens, Athens 12462; \\ ${ }^{3}$ First Department of Surgery, Laiko General Hospital; ${ }^{4}$ Laboratory of Experimental Surgery and Surgical Research, \\ National and Kapodistrian University of Athens, Athens 11527, Greece
}

Received November 14, 2019; Accepted March 3, 2020

DOI: 10.3892/br.2020.1295

\begin{abstract}
Defects in the Canal of Nuck are rare abnormalities of the female genitalia, which are typically detected and repaired in young age. In the present report, a case of a Nuck cyst in a 40-year old female patient is described. Additionally, the current literature concerning cases of women with hydrocele of Nuck canal was systematically reviewed. A total of 16 case reports of 16 patients with Nuck hydrocele (mean age of 35.18 years), have been reported to date. A right inguinal mass was noted in 13 patients $(81.3 \%)$ whereas in 3 patients a left-sided mass was noted. The surgical approach was open in 13 cases and laparoscopic in 3 cases. Two cases underwent hydrocelectomy and inguinal ring ligation, whereas in 7 cases a simple cystectomy was performed. In 2 cases the round ligament was excised along with the hydrocele. In one of these 2, ligamentum rotundum necrosis and presence of a haemorrhagic cyst of the canal of Nuck were identified. Hernia repair and hydrocelectomy was performed in 5 cases. A cyst of the Canal of Nuck is relatively rare, but should be considered during the diagnosis of inguinal masses in female patients.
\end{abstract}

\section{Introduction}

Defects in the canal of Nuck are rare abnormalities of the female genitalia that are usually detected and repaired in young girls more frequently during the first 5 years of life (1). The first noted case defect in the Canal of Nuck dates back to 1691 by Anton Nuck (2). The failure of the canal to close after birth or within the first year of life in female infants can lead to formation of hydrocele or herniation of intraabdominal

Correspondence to: Dr Anastasia Prodromidou, Department of Surgery, Division of Surgical Oncology, Metaxa Cancer Memorial Hospital, Mpotasi 51, Piraeus 18573, Greece

E-mail: a.prodromidou@hotmail.com

Key words: hydrocele, Nuck cyst, Canal of Nuck, inguinal swelling, groin mass structures through the patent Canal of Nuck $(1,3)$. Hydrocele of the Nuck canal, cyst of the Canal of Nuck or female hydrocele are equivalent terms for a rare developmental disorder of the reproductive system of women and accounts for a limited number of cases of benign painless or painful swelling in the inguinal region or even to the labia majora $(4,5)$. Hydrocele of the Canal of Nuck constitutes a particular type of primary idiopathic hydrocele, the enlargement of which has been attributed to a defect of the secretory membranes resulting in an imbalance in secretion and absorption of fluids of the processus vaginalis (6).

Several reports of cysts of the Canal of Nuck are presented in literature and describe the symptomatology and surgical approaches used in their treatment (7-10). Although a rare pathology of the inguinal canal in female patients, it should be considered when diagnosing inguinal tumours in female patients. In the present report, a case of cyst of Nuck in a female patient, and a review of the current literature with special consideration on clinical presentation, diagnostic approach and management are presented.

\section{Case presentation}

A 40-year old female presented to our department with a painful mass in her right inguinal region. The swelling was first noticed two months ago, and the patient reported she never suffered from regional pain before. There was no history of local trauma, symptoms of nausea, vomiting or abdominal discomfort. Her body mass index was $27 \mathrm{~kg} / \mathrm{m}^{2}$. Her medical history was negative for any pathology and surgical procedures. She had two uncomplicated vaginal deliveries. At presentation, physical examination revealed a small palpable mobile lump in the right groin without overlying skin erythema or tenderness. Valsalva maneuver did not make the mass more prominent. There was an absence of incarceration or strangulation. Her abdomen was soft, non-distended and non-tender with no signs of bowel occlusion. Laboratory tests showed measured parameters were within the normal range. Magnetic resonance imaging (MRI) revealed a $3.5 \mathrm{~cm}$ well-defined, thin-walled cystic structure in her right groin (Fig. 1). There was no evidence of bowel loops, omentum or other solid structures 
within the mass. The diagnosis of a Nuck cyst was considered and the patient was operated on. The cyst was dissected from the round ligament and was completely excised. The defect of the internal inguinal ring was primarily repaired without the use of a mesh. Histology confirmed the presence of Nuck cyst. The patient's postoperative course was uneventful, and she was discharged the next day. One year postoperatively, the patient remained asymptomatic without any recurrence. Written informed consent was obtained from the patient for publication of this case report and any accompanying images.

\section{Review of the literature}

A systematic search of the literature for articles published between January 2000 and May 2019 was performed using the Medline (1966-2019), Scopus (2004-2019) and Google Scholar (2004-2019) databases along with the references in any articles. For all articles, the full-text was retrieved. Studies regarding adult women who were diagnosed with Nuck hydrocele were considered eligible for inclusion. A total of five studies were excluded from the present review (11-15). Pandey et al (11) and Safak et al (12) presented radiological outcomes of a case with Nuck hydrocele and thus excluded due to insufficient data. Noguchi et al (13) and Amu et al (14) were not considered eligible due the fact that the cystic structure in the Nuck canal was histologically confirmed as ectopic pregnancy and dermoid cyst, respectively, and not as hydrocele. Finally, Sala et al (15) included a 17 -year old patient with Nuck cyst and was excluded due to age restrictions of the present study.

A total of 16 case reports of 16 patients with hydrocele of the Canal of Nuck, were included in the present systematic review (3-5,7-10,16-24); whereas 5 studies were excluded. The primary characteristics of the included studies including the primary symptoms on presentation, outcomes of clinical examination and imaging, the size of the mass and the type of procedure performed are presented in Table I. The mean age of patients included was $35.18 \pm 3.27$ years and $13(81.3 \%)$ of the patients were of reproductive age (18-45 years). Two of the included women were nulliparous; one of them presented with infertility and underwent simultaneous surgery for an enlarged ovarian cyst. Three women had one or more children. For the remaining 11 patients, data concerning parity was not available. A right inguinal mass was noted in the majority of the patients $(n=13,81.3 \%)$ whereas in the remaining thee patients a left-sided mass was noted. The enrolled patients were admitted with a groin swelling which was either painful in 5/16 cases or painless in the remaining 11/16 cases. In all except one case, preoperative imaging was performed; In six patients an ultrasound (US) was performed, preoperative computed tomography (CT) was performed in three cases, one patient underwent MRI, in one case a CT along with an MRI was performed, in four cases US and MRI were performed, and in one case US in combination with CT was performed. In seven cases, imaging suggested the presence of a cystic structure suggestive of the presence of hydrocele of the Canal of Nuck. Clinical examination revealed a reducible lump in the affected inguinal region in six women, and in eight cases, the mass was irreducible. In two cases, the nature of the mass was not specified.

All the included patients underwent surgery to examine the lesion and repair the defect. In 13 patients the surgical
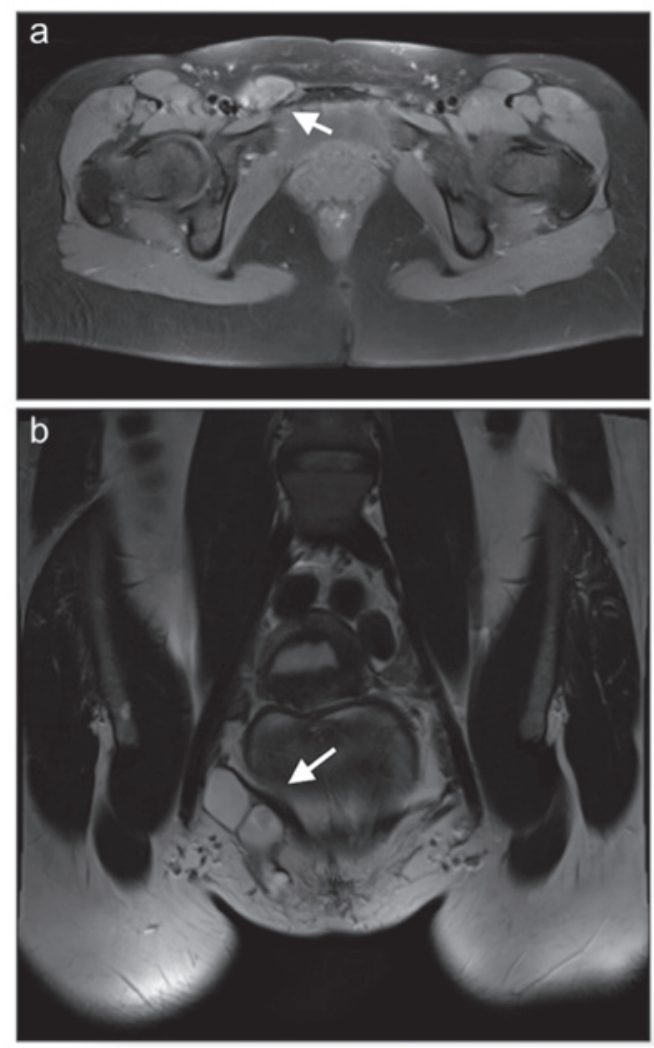

Figure 1. Magnetic resonance imaging showing a thin-walled cystic structure located in the right groin. (a) Axial view. (b) Coronal view.

approach was open, and the other three patients underwent laparoscopy. Two cases underwent excision of the hydrocele and inguinal ring ligation, and in six cases a simple cystectomy was performed, which was followed by a vulva correction in one case. In 2 cases the round ligament was excised, with the hydrocele in one of them due to necrosis and presence of a haemorrhagic cyst. A hematoma within a Nuck hydrocele was found in one case and evacuated along with the hydrocelectomy. Hernia repair along with hydrocelectomy was performed in five cases (three laparoscopic and two open); among which, in two cases, a herniorrhaphy (no mesh repair) was performed, whereas three patients underwent a hernia repair with the use of a mesh. An additional inguinal ring ligation was performed in two patients; one laparoscopic and one open. Postoperative recovery was uneventful, and no postoperative deaths or complications were observed. According to 7 of the included studies, no recurrence of the lesion was reported in the follow up period, which ranged from 2-24 months after surgery $(3,8,9,16,18,20,22)$. Data concerning hospital stay was reported in 4 studies with a range of 20-72 $\mathrm{h}(7,10,18,20)$.

\section{Discussion}

The Canal of Nuck runs through the inguinal canal adjacent to the round ligament and is considered the female analogue of the processus vaginalis in males (25). Normally, the Canal of Nuck is obliterated within the first year of life. Failure of the Canal to close during that period in female infants can result in Nuck hydrocele or herniation of intraabdominal structures through the patent Canal of Nuck (1). Thus, failure of closure is typically 


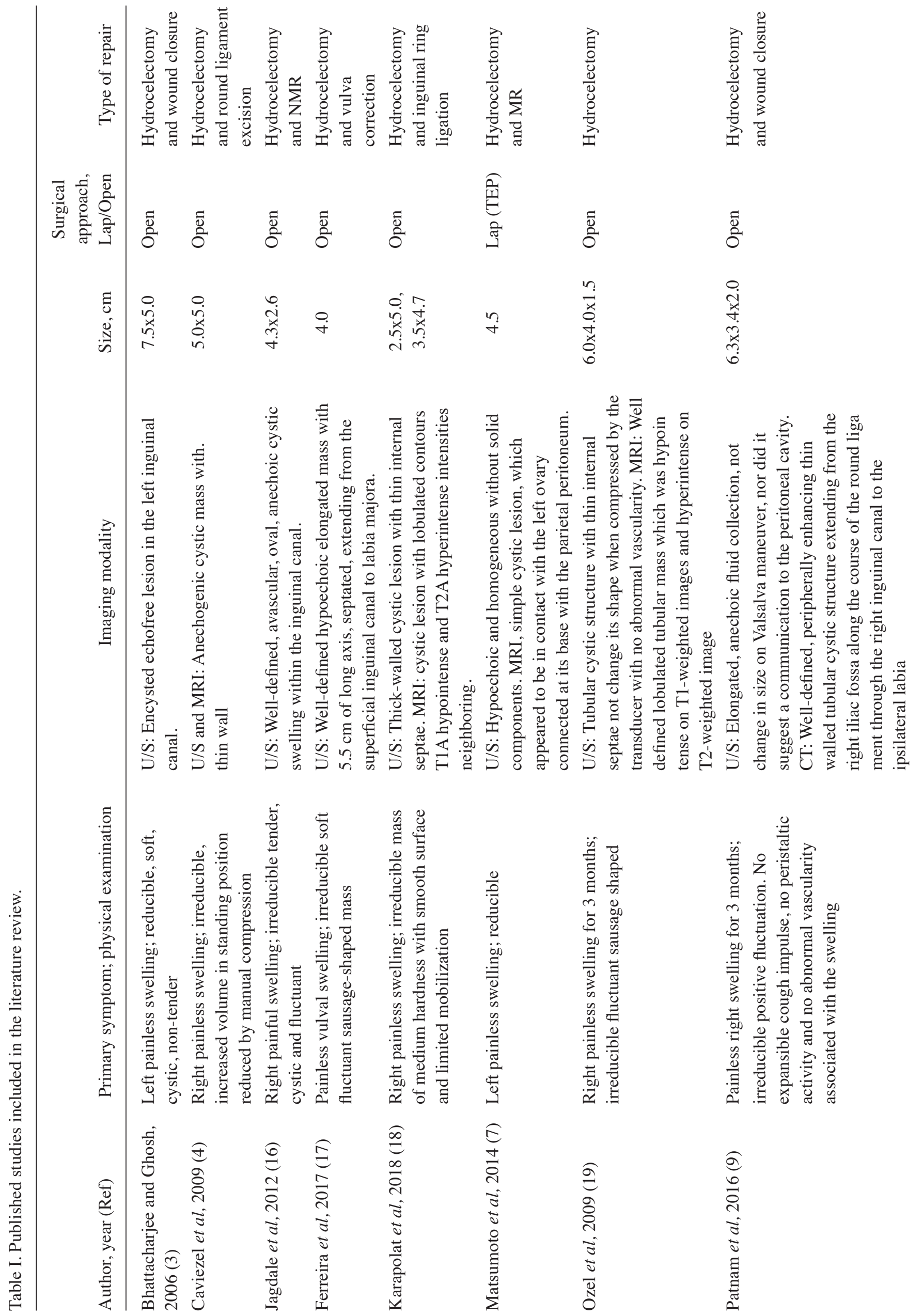




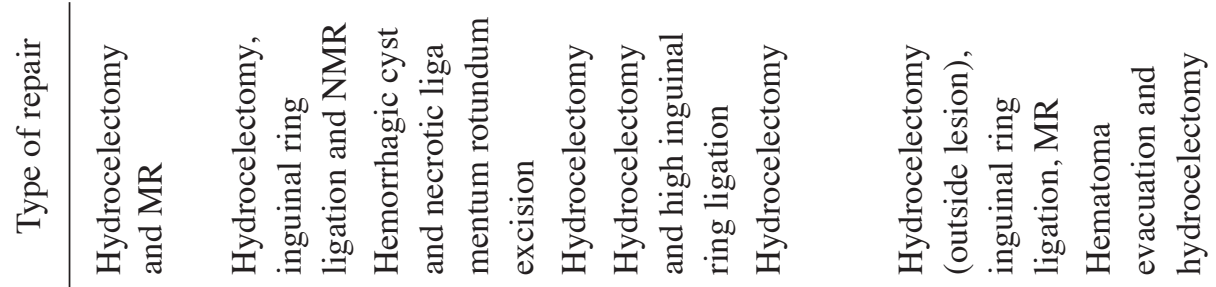

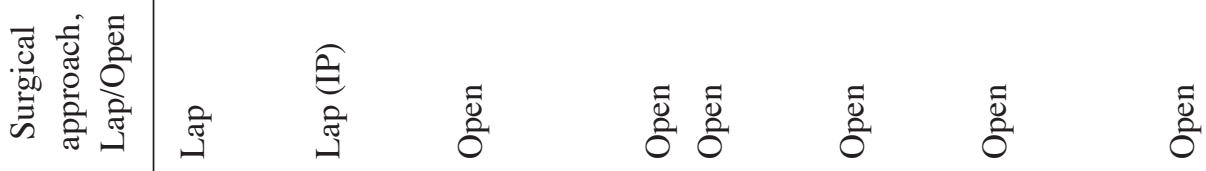

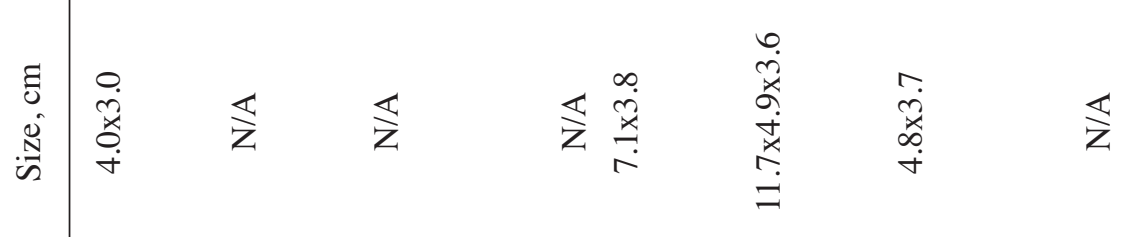

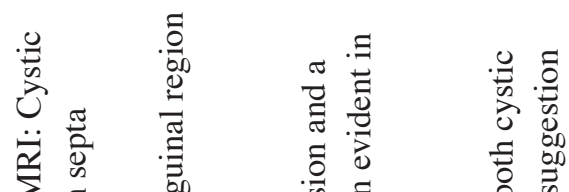

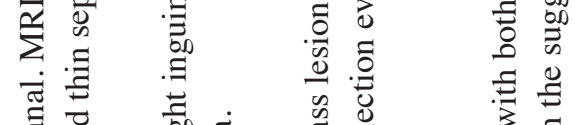

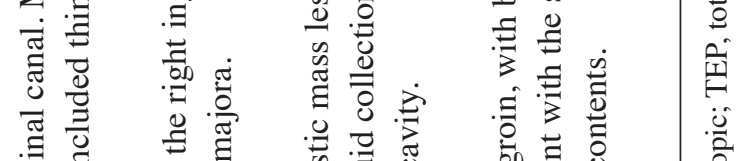

를

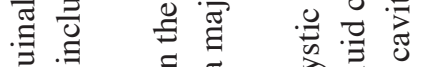

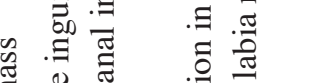

उ.

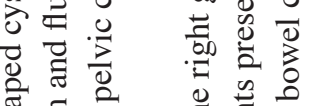

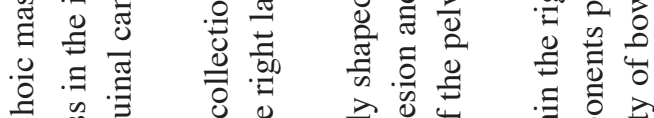

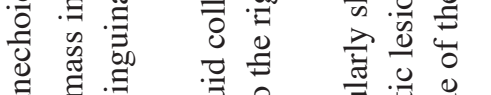

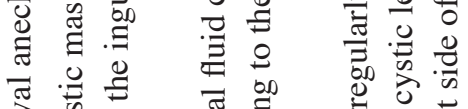

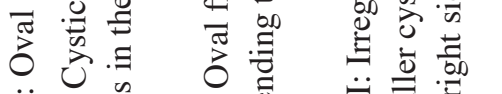

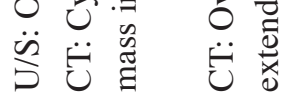

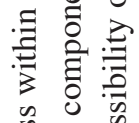

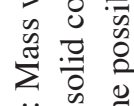

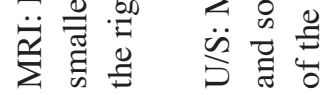

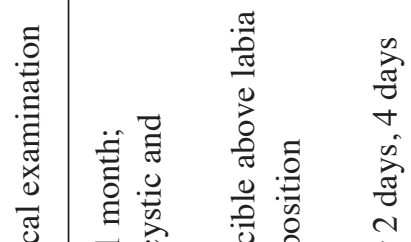

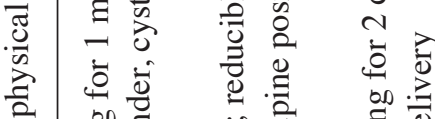

ล.

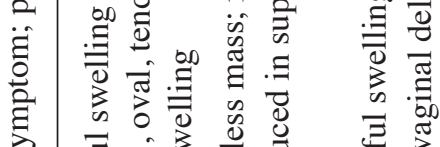

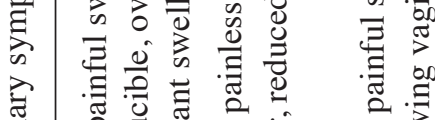

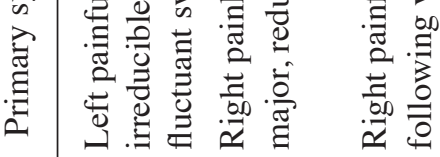

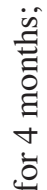

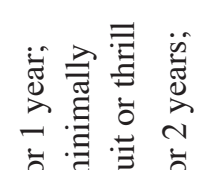

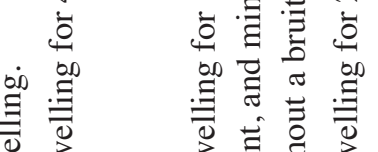

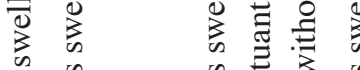

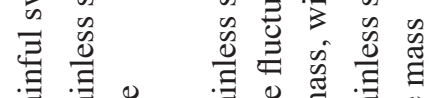

吾离

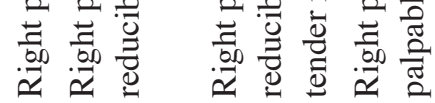

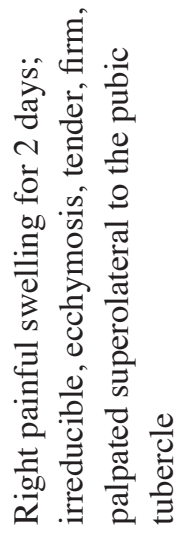

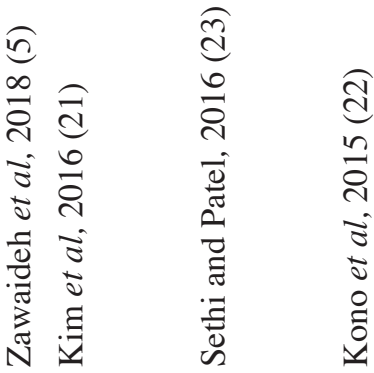

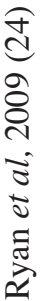




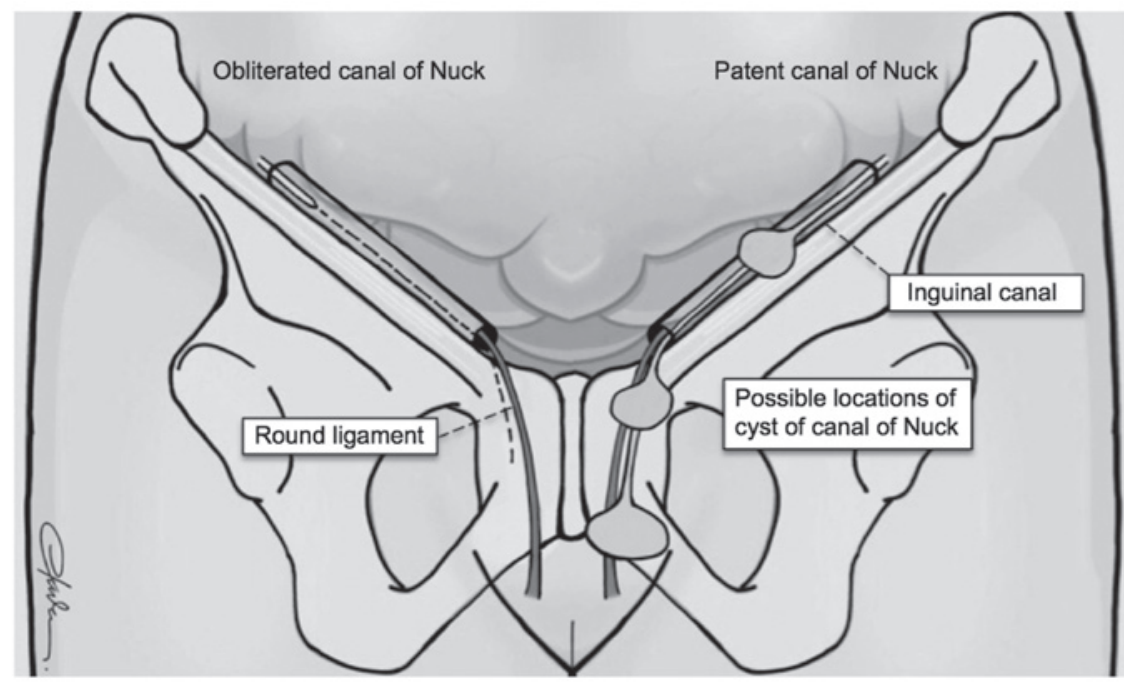

Figure 2. Anatomy of the inguinal canal with a physiologically obliterated Canal of Nuck and the potential sites of the cyst through the canal when it remains patent.

detected in childhood. Due to its rarity, accurate information regarding the exact prevalence of hydrocele during childhood is not available. Akkoyun et al (26) reported that only $0.76 \%$ of girls $<12$ years exhibited hydrocele of Nuck among their study population. A comparable prevalence of Nuck hydrocele $(0.74 \%)$ was also reported by Papparella et al (27), who reviewed 353 female patients, aged 1-14 years, with inguinal swelling. Literature regarding Nuck hydrocele in adulthood are even more scarce. A systematic review performed in the present study revealed a total of 16 cases of adult patients who underwent surgery for Nuck cyst.

Fig. 2 schematically presents the anatomy of the inguinal canal with a physiologically obliterated Canal of Nuck and the potential sites where the cyst may be recognized when the canal is patent. At clinical examination, the cyst of the Canal of Nuck is frequently described as a painless or mildly painful irreducible or reducible mass in the inguinal region, which typically extends to the labia majora, and does not expand when performing the Valsalva manoeuvre (23). In approximately one-third of patients an associated inguinal hernia is present. Consistent with this, nearly one-third of the patients included in the present study underwent a simultaneous hernia repair. Differential diagnosis includes inguinal hernia, enlarged lymph nodes and soft tissue tumours such as lipomas, leiomyomas and endometriosis of the round ligament (28).

A cyst of the Canal of Nuck is frequently misdiagnosed as inguinal hernia in females and is only correctly diagnosed intraoperatively. Therefore preoperative imaging is crucial for facilitating diagnosis and further guiding therapeutic options. All except one of the included cases included in the systematic review underwent preoperative imaging with US, CT, MRI, or a combination of these. High-resolution real-time sonography serves as an inexpensive and accurate modality for differentiating hydrocele of the Canal of Nuck from the aforementioned conditions. In an ultrasound scan, Nuck cyst appears as a thin walled, tubular or dumbbell shaped, well defined, anechoic or hypoechoic, unilocular or multilocular cystic structure $(19,22)$. In a colour Doppler scan, the Nuck cyst does not show any internal vascularity $(28,29)$. Additionally, an MRI may also be performed, particularly in complicated cases, such as those involving additional pathologies where hydrocele usually appears as a well-defined, thin-walled cystic lesion in hypointense on T1-weighted and hyperintense on T2-weighted series (1). Imaging with MRI allows for good visualization of the anatomic structures surrounding the cyst, communication between the cyst and the peritoneal cavity and the extension of the cyst of the Canal of Nuck (22). However, despite the utility of imaging in differential diagnosis, surgery along with histological and immunohistological analysis of the excised mass is required for a more conclusive diagnosis of a Nuck cyst.

Another issue that should be addressed is the association of the pathology of the Canal of Nuck with fertility. In the present case report as well as in the published literature, there was only one association with infertility; a nulliparous woman of reproductive age who underwent simultaneous ovarian cyst excision and repair of patent Canal of Nuck (20). Nonetheless, infertility in this case was not attributed to the Nuck canal pathology. Postoperative courses of all published cases are uneventful. Unfortunately, data concerning postoperative fertility was not available for any of the reported cases. Other pathologies of the Canal of Nuck such as ovary herniation or endometriosis of the canal may result in infertility in young females (30).

Surgical management of a cyst of the Canal of Nuck includes open or laparoscopic excision of the cystic structure with concomitant closure of the inguinal internal defect primarily with the use of a mesh $(24,31,32)$. The appropriate surgical approach is tailored based on the extent of the disease, the accuracy of preoperative diagnosis and the co-existence of an inguinal hernia. In the case of concomitant identification of an inguinal hernia, an additional hernia repair with or without mesh placement can be safely performed. Furthermore, as described by Ferreira et al (17), an additional vulva correction may be indicated in cases of mass extension to the labia majora.

To the best of our knowledge this is the first literature review which presents a cumulative report of cases of adult females with Nuck cyst. The risk of potential loss of relevant literature was eliminated by performing a thorough search of the current literature. Due to limited data from case reports and 
small case series, the actual prevalence of Nuck cyst could not be precisely estimated. The significant heterogeneity among the included studies along with the lack of mention of certain parameters by some authors were additional limitations. In conclusion, the cyst of the canal of Nuck is a rare condition, but it should be included in the differential diagnosis list of inguinal tumours in female patients.

\section{Acknowledgements}

Not applicable.

\section{Funding}

No funding was received.

\section{Availability of data and materials}

The datasets used and/or analysed during the present study are available from the corresponding author on reasonable request.

\section{Authors' contributions}

APr and NM conceived and designed the study. APr, APa, DS, $\mathrm{CN}$ and ES acquired, analysed and interpreted the data. APr, $\mathrm{APa}, \mathrm{DS}, \mathrm{CN}, \mathrm{NM}$ and ES drafted and revised the manuscript. All authors read and approved the final manuscript.

\section{Ethics approval and consent to participate}

Not applicable.

\section{Patient consent for publication}

Written informed consent was obtained from the patient for publication of this case report and any accompanying images.

\section{Competing interests}

The authors declare that they have no competing interests.

\section{References}

1. Rees MA, Squires JE, Tadros S and Squires JH: Canal of Nuck hernia: A multimodality imaging review. Pediatr Radiol 47 893-898, 2017.

2. Tubbs RS, Loukas M, Shoja MM, Salter EG and Oakes WJ: Indirect inguinal hernia of the urinary bladder through a persistent canal of Nuck: Case report. Hernia 11: 287-288, 2007.

3. Bhattacharjee PK and Ghosh G: Hydrocele of the canal of Nuck. J Indian Med Assoc 104: 150-151, 2006.

4. Caviezel A, Montet X, Schwartz J, Egger JF and Iselin CE: Female hydrocele: The cyst of Nuck. Urol Int 82: 242-245, 2009.

5. Zawaideh JP, Trambaiolo Antonelli C, Massarotti C, Remorgida V and Derchi LE: Cyst of Nuck: A disregarded pathology. J Minim Invasive Gynecol 25: 376-377, 2018.

6. Dagur G, Gandhi J, Suh Y, Weissbart S, Sheynkin YR, Smith NL, Joshi G and Khan SA: Classifying hydroceles of the pelvis and groin: An overview of etiology, secondary complications, evaluation, and management. Curr Urol 10: 1-14, 2017.

7. Matsumoto T, Hara T, Hirashita T, Kubo N, Hiroshige S and Orita $\mathrm{H}$ : Laparoscopic diagnosis and treatment of a hydrocele of the canal of Nuck extending in the retroperitoneal space: A case report. Int J Surg Case Rep 5: 861-864, 2014.

8. Qureshi NJ and Lakshman K: Laparoscopic excision of cyst of canal of Nuck. J Minim Access Surg 10: 87-89, 2014.
9. Patnam V, Narayanan R and Kudva A: A cautionary approach to adult female groin swelling: Hydrocoele of the canal of Nuck with a review of the literature. BMJ Case Rep 2016: pii: bcr2015212547, 2016.

10. Uzun I, Inan C, Varol F, Erzincan S, Sutcu H and Sayin C: Hemorrhagic cyst of the canal of Nuck after vaginal delivery presenting as a painful inguinal mass in the early postpartum period. Eur J Obstet Gynecol Reprod Biol 213: 147-148, 2017.

11. Pandey A, Jain S, Verma A, Jain M, Srivastava A and Shukla RC: Hydrocele of the canal of Nuck-Rare differential for vulval swelling. Indian J Radiol Imaging 24: 175-177, 2014.

12. Safak AA, Erdogmus B, Yazici B and Gokgoz AT: Hydrocele of the canal of Nuck: Sonographic and MRI appearances. J Clin Ultrasound 35: 531-532, 2007.

13. Noguchi D, Matsumoto N, Kamata S and Kaneko K: Ectopic pregnancy developing in a cyst of the canal of Nuck. Obstet Gynecol 123: 472-476, 2014.

14. Amu OC, Udeh EI, Ugochukwu AI, Madu C and Nzegwu MA: A case of vulval swelling secondary to female circumcision posing a diagnostic dilemma. Int J Surg Case Rep 3: 431-434, 2012.

15. Sala P, Palmeri A and Costantini S: Giant cyst of the Nuck canal: A worrisome trouble for a girl. Am J Obstet Gynecol 218: 530-531, 2018.

16. Jagdale R, Agrawal S, Chhabra S and Jewan SY: Hydrocele of the canal of Nuck: Value of radiological diagnosis. J Radiol Case Rep 6: 18-22, 2012.

17. Ferreira AF, Marques JP and Falcao F: Hydrocele of the canal of Nuck presenting as a sausage-shaped mass. BMJ Case Rep 2017: pii: bcr-2017-221024, 2017.

18. Karapolat B, Ata Korkmaz HA, Kocak G and Bulut E: Image of the month: Cyst of the canal of Nuck. Acta Chir Belg 118: 138-140, 2018.

19. Ozel A, Kirdar O, Halefoglu AM, Erturk SM, Karpat Z, Lo Russo G, Maldur V and Cantisani V: Cysts of the canal of Nuck: Ultrasound and magnetic resonance imaging findings. J Ultrasound 12: 125-127, 2009.

20. Yen CF, Wang CJ, Chang PC, Lee CL and Soong YK: Concomitant closure of patent canal of Nuck during laparoscopic surgery: Case report. Hum Reprod 16: 357-359, 2001.

21. Kim KS, Choi JH, Kim HM, Kim KP, Kwon YJ, Hwang JH and Lee SY: Hydrocele of the canal of nuck in a female adult. Arch Plast Surg 43: 476-478, 2016.

22. Kono R, Terasaki H, Murakami N, Tanaka M, Takeda J and Abe T: Hydrocele of the canal of Nuck: A case report with magnetic resonance hydrography findings. Surg Case Rep 1: 86, 2015.

23. Sethi V and Patel H: Hydrocele in the Canal of Nuck-CT appearance of a developmental groin anomaly. J Radiol Case Rep 10: 29-33, 2016.

24. Ryan JD, Joyce MR, Pierce C, Brannigan A and O'Connell PR: Haematoma in a hydrocele of the canal of Nuck mimicking a Richter's hernia. Hernia 13: 643-645, 2009.

25. Choi YM, Lee GM, Yi JB, Yoon KL, Shim KS, Bae CW, Choi SI and Kim HC: Two cases of female hydrocele of the canal of nuck. Korean J Pediatr 55: 143-146, 2012.

26. Akkoyun I, Kucukosmanoglu I and Yalinkilinc E: Cyst of the canal of nuck in pediatric patients. N Am J Med Sci 5: 353-356, 2013.

27. Papparella A, Vaccaro S, Accardo M, De Rosa L, Ronchi A and Noviello C: Nuck cyst: A rare cause of inguinal swelling in infancy. Minerva Pediatr: Jul 23, 2018 (Epub ahead of print).

28. Stickel WH and Manner M: Female hydrocele (cyst of the canal of Nuck): Sonographic appearance of a rare and little-known disorder. J Ultrasound Med 23: 429-432, 2004.

29. Park SJ, Lee HK, Hong HS, Kim HC, Kim DH, Park JS and Shin EJ: Hydrocele of the canal of Nuck in a girl: Ultrasound and MR appearance. Br J Radiol 77: 243-244, 2004.

30. Choi KH and Baek HJ: Incarcerated ovarian herniation of the canal of Nuck in a female infant: Ultrasonographic findings and review of literature. Ann Med Surg (Lond) 9: 38-40, 2016.

31. Bagul A, Jones S, Dundas S and Aly EH: Endometriosis in the canal of Nuck hydrocele: An unusual presentation. Int J Surg Case Rep 2: 288-289, 2011.

32. Jimenez JS, Barbero P, Tejerizo A, Guillen C and Strate C: A laparoscopic approach to Nuck's duct endometriosis. Fertil Steril 96: e103-e105, 2011. 